\title{
Observation of light echoes around very young stars ${ }^{\star}$
}

\author{
J. L. Ortiz ${ }^{1}$, B. E. K. Sugerman ${ }^{2}$, I. de la Cueva ${ }^{3}$, P. Santos-Sanz ${ }^{1,4}$, R. Duffard ${ }^{1}$, R. Gil-Hutton ${ }^{5}$,
} M. Melita ${ }^{6}$, and N. Morales ${ }^{1}$

1 Instituto de Astrofísica de Andalucía - CSIC, Apt 3004, 18080 Granada, Spain e-mail: duffard@iaa.es

2 Department of Physics and Astronomy, Goucher College, 1021 Dulaney Valley Rd., Baltimore, Maryland 21204, USA

3 Astroimagen. C. Abad y Lasierra, 58 Bis - 62, 07800 Ibiza, Islas Baleares, Spain

${ }^{4}$ Observatoire de Paris, LESIA-UMR CNRS 8109, 5 place Jules Janssen, 92195 Meudon Cedex, France

5 Complejo Astronómico El Leoncito (CASLEO-CONICET) and San Juan National University, Avda. de España 1512 sur, J5402DSP, San Juan, Argentina

${ }^{6}$ Instituto de Astronomía y Física del Espacio, Universidad de Buenos Aires, CONICET, CC 67-Suc 28, C1428ZAA, Ciudad Autónoma de Buenos Aires, Argentina

Received 16 March 2010 / Accepted 1 June 2010

\section{ABSTRACT}

\begin{abstract}
Aims. The goal of the paper is to present new results on light echoes from young stellar objects.
Methods. Broad band CCD images were obtained over three months at one-to-two week intervals for the field of NGC 6726, using the large field-of-view remotely-operated telescope on top of Cerro Burek.

Results. We detected scattered light echoes around two young, low-amplitude, irregular variable stars. Observations revealed not just one, but multiple light echoes from brightness pulses of the T Tauri star S CrA and the Herbig Ae/Be star R CrA. Analysis of $\mathrm{S}$ CrA's recurring echoes suggests that the star is located $138 \pm 16 \mathrm{pc}$ from Earth, making these the closest echoes ever detected. The environment that scatters the stellar light from S CrA is compatible with an incomplete dust shell or an inclined torus some $10000 \mathrm{AU}$ in radius and containing $\sim 2 \times 10^{-3} M_{\odot}$ of dust. The cause of such concentration at $\sim 10000 \mathrm{AU}$ from the star is unknown. It could be the remnant of the envelope from which the star formed, but the distance of the cloud is remarkably similar to the nominal distance of the Oort cloud to the Sun, leading us to also speculate that the dust (or ice) seen around S CrA might have the same origin as the Solar System Oort cloud.
\end{abstract}

Key words. stars: protostars - stars: variables: T Tauri, Herbig Ae/Be - stars: imaging

\section{Introduction}

Light echoes can be produced when the light pulse from an astronomical source is scattered by dust. If the direction of the scattering is toward the Earth and the pulse is intense enough, an echo can be observed from Earth (Couderc 1939), provided that the number density of dust particles is high enough. All those conditions are not usually met. In fact, light echoes are impressive and very rare phenomena that have been witnessed in only a handful of events (Couderc 1939; Suntzeff et al. 1988; Bode \& Evans 1985; Rest et al. 2005; Krause et al. 2005; Bond et al. 2003; Sugerman 2005; Quinn et al. 2006; Sugerman 2003; Crotts \& Yourdon 2008; Liu et al. 2003; Rest et al. 2008a,b; Cappellaro et al. 2001; Sparks et al. 1999, 2008; Bond \& Sparks 2009, and references therein). Such events are mostly explosive or nearly explosive (supernovae, novae, novae-like, a Cepheid variable, and a planetary nebula) from stellar objects in their latest stages of evolution.

Since scattered-light echoes reveal the 3-dimensional structure and chemical composition of dusty circumstellar and interstellar environments around variable sources, they should serve as powerful probes of early stages of star and planet formation,

* Movies are available in electronic form at http: //www . aanda. org,

http://www.iaa.es/ ortiz/animacion1.avi, and http://www.iaa.es/ ortiz/S-animation.gif when the young stellar sources exhibit irregular, low-amplitude variability. Thus, finding and analyzing echoes from young stellar sources would be a powerful tool to study stellar and planetary formation. Although theoretical predictions exist for the detectability of echoes around young variable stars (e.g. Sugerman 2003; Gaidos 1994), systematic searches have not been carried out, perhaps because the low detection rate from intentional searches around supernovae (Boffi et al. 1999) has suggested that fainter echoes are especially difficult to observe.

However, with current and suitable technology, faint echoes from young stars can be observed. Here we present the detection of multiple light echoes from two young variable stars, the Herbig $\mathrm{Ae} / \mathrm{Be}$ star $\mathrm{R} \mathrm{CrA}$ and $\mathrm{T}$ Tauri star S CrA. It must be mentioned that for $\mathrm{R}$ CrA, short-term variability in the brightness of its nebulosity (NGC 6729) has been reported before (e.g. Hubble 1921, Graham \& Phillips 1989), and also similar changes have been reported in other variable nebulae like NGC 2261 (e.g. Hubble 1921; Lightfoot 1989) and in NGC 1555. The question of light variability in nebulae was a key issue at the end of the 19th century, and there are reports going back to 1862 (Struve 1862) about the variability of NGC 1555. A somewhat newer account of light variability in the complex surroundings of T Tauri is given by Barnard (1895).

After a careful reading of Hubble reports (1921) on several variable nebulae, a phenomenon of the expansion of a bright envelope around R CrA (of just a few arcsecs in a few days), 
Table 1. Observing Log.

\begin{tabular}{lccccc}
\hline \hline Date & UT & Exp time $(\mathrm{s})$ & $V \mathrm{Mag}^{a}$ & Angular distance $^{b}$ & Surface brightness $^{c}$ \\
\hline 2454298.62 & $2007071702: 49$ & 1800 & 11.1 & 45 & 2.0 \\
2454325.59 & $2007081302: 12$ & 1800 & 10.7 & 20 & 1.2 \\
2454328.57 & $2007081601: 46$ & 1800 & 11.1 & 30 & 2.7 \\
2454332.54 & $2007082000: 57$ & 1800 & 11.1 & 40 & 1.6 \\
2454352.56 & $2007090901: 33$ & 2400 & 11.5 & 55 & 2.0 \\
2454356.52 & $2007091300: 34$ & 2400 & 11.7 & 60 & 0.7 \\
2454361.58 & $2007091801: 52$ & 3000 & 11.4 & 65 & 5.0 \\
2454384.52 & $2007101100: 32$ & 2400 & 10.9 & 35 & 3.4 \\
2454390.52 & $2007101700: 38$ & 3000 & 11.2 & 45 & \\
\hline
\end{tabular}

Notes. ${ }^{(a)}$ Typical uncertainties are $0.2 \mathrm{mag}$; ${ }^{(b)}$ given in $\operatorname{arcsec}$; typical uncertainties are $2^{\prime \prime}$; ${ }^{(c)}$ given in units of $10^{-19} \mathrm{erg} \mathrm{cm}^{-2} \mathrm{~s}^{-1} \AA^{-1} \operatorname{arcsec}^{-2}$; typical uncertainties are $10^{-20} \mathrm{erg} \mathrm{cm}^{-2} \mathrm{~s}^{-1} \AA^{-1} \operatorname{arcsec}^{-2}$.

which is called in the report "... a wave of illumination moving..." qualifies as a true light echo, although it was not realized as such at the time. On the other hand, the derivation of the distance to the star made by Hubble was based on incorrect analysis, like the analyses of the Nova Per (1901) light echo prior to Couderc's (1939) correct interpretation of the light echo phenomenon. Maybe those are the reasons the R CrA inner core expansion phenomenon has never been recognized in the light echo literature so is not listed in any light echo compilation. Regardless of when the first detection of a light echo around a young star took place, it must be pointed out that the R CrA light echoes presented here are much farther away from the star than "the wave of illumination" reported in Hubble (1921) and farther away than the variability areas reported in previous works (e.g. Hubble 1921; Bellingham \& Rossano 1980; Graham \& Phillips 1987). Concerning the T Tauri S CrA, no variability in its nebula has ever been reported and the finding of its light echo is reported here for the first time. In this paper we focus on a preliminary analysis of the echoes surrounding S CrA.

\section{Observations and reductions}

As part of a southern-hemisphere sky survey carried out at the Complejo Astronómico El Leoncito (CASLEO) in Argentina, a field containing the reflection nebula NGC 6726 was imaged twice, with roughly one month between observations. The observations were made by means of our large field-of-view $0.45 \mathrm{~m} \mathrm{f} / 2.8$ remotely-operated telescope on top of Cerro Burek, through a broad (390 to $700 \mathrm{~nm}$ ) filter. The detector was a $4008 \times$ 2672 pixel camera based on the Kodak KAF11000M CCD chip. The pixel scale of the setup was 1.47 arcsec/pixel. In the course of standard reduction and analysis, the very young stars S CrA and $\mathrm{R} \mathrm{CrA}$ experienced significant brightness changes. Closer inspection of the data clearly revealed material that appeared to be expanding away from the two stars at superluminal speeds for all plausible values of the distances to the stars. The appearance of such superluminal motion is well understood as the scattering of a light pulse off of stationary dust near the line of sight, rather than physical expansion of material ejected by the star (Couderc 1939). Light echoes not only illuminate otherwise hidden dust, but also directly reveal that material's 3-dimensional structure. Since mapping the circumstellar environments of such young stars has the potential to reveal critical details of their formation processes, we re-imaged the field of NGC 6726 over the next three months at one-to-two week intervals, as listed in Table 1. Each image was reduced in the standard manner (e.g. bias subtraction, flat fielding), then geometrically registered to a common orientation using field stars as described in Sugerman (2005). rms residuals of geometric registration are less than 0.1 pixel for all images. Finally, data were flux calibrated to the $V$-band $( \pm 0.1 \mathrm{mag})$ using field stars from the UCAC2 catalog (Zacharias et al. 2004).

Figure 1 shows the reflection nebula NGC 6726 and surrounding field, with the locations of $\mathrm{S}$ and $\mathrm{R} \mathrm{CrA}$ indicated. A full color animation of that field is provided as supplementary online material. The time-variable light echoes are immediately obvious in the animation as they move away from their sources. A temporal sequence of the field immediately surrounding S CrA is shown in Fig. 2. The echoes can also be faintly seen in the direct images (left column of Fig. 2), and are slightly more apparent when the point-spread function (PSF) of S CrA has been removed (Stetson 1987), as shown in the middle column. Since light echoes are transient phenomena, we employed the PSF-matching and difference-imaging techniques of Tomaney \& Crotts (1996) and Sugerman (2005), combined with the NN2 algorithm of Newman \& Rest (2006), to remove all sources of constant flux and produce echo-only images for each epoch. These are shown in the right column of Fig. 2.

An animated close-up view of S CrA subtracted images in which the residuals from the subtractions have been removed by interpolation is shown as online material. Expanding concentric rings or "quasi-rings" are clearly visible in this animation. As revealed by the animation and in Fig. 2, the echoes have an overall circular morphology but the circles are incomplete; that is why the term "quasi-rings" would be more appropriate to describe them. These expanding echoes are analyzed in the next section.

\section{S CrA echo interpretation}

In the typical approach to model nova or supernovae echoes, the light curve and distance of the source are used to deduce the 3-dimensional structure of the dust producing the echoes (Sugerman 2003). Such an approach is not feasible with our S CrA data. S CrA has been observed as part of the All Sky Automated Survey (Pojmanski 1997), however the photometry (Fig. 3) does not have homogenous time sampling, making the epochs of maximum light and the pulse durations moderately uncertain. Additionally, there are few distance measurements to the star, with the best estimates ranging from 120 to $140 \mathrm{pc}$ (Marraco \& Rydgren 1981; Carmona et al. 2007), as there are no reliable parallax measurements from Hipparcos (see the discussion on this topic in Neuhäuser \& Forbrich 2008). In such circumstances, we instead constrain the above properties by comparing the echoes that would arise from a test dust geometry to those actually observed. We initially use guesses for the dates and times of four outbursts and later on we refine such estimates based on the observed light curve. A minimum of 4 outbursts are needed for the following reason. While we see echoes expanding 


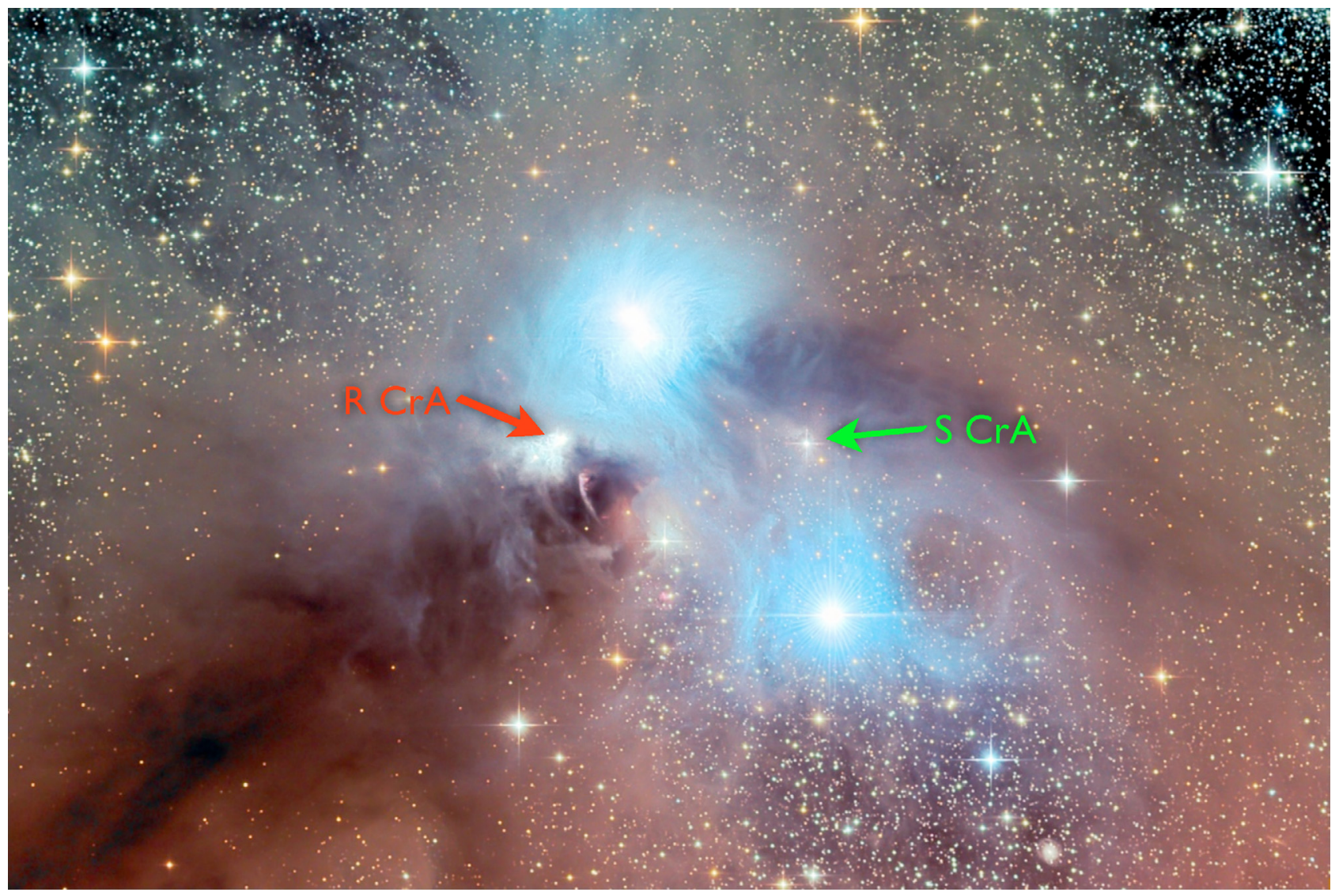

Fig. 1. Color composite assembled from $B, V$, and $R$ images of a $47 \times 31$ arcmin field of view of the reflection nebula NGC 6726 , with the positions of R CrA and S CrA indicated. Astronomical north is up and east is left. An animation showing a $30.3 \times 22.1$ arcmin enlargement of the full temporal sequence of observations (Table 1) is available online. Note in the animation that time-variable signals are centered on both $\mathrm{S}$ CrA and $\mathrm{R} \mathrm{CrA}$, and that the animation works best when viewed as a continuous loop.

in the first set of observations, a month later on we see echoes of smaller angular size, which means that new echoes have been formed; thus another pulse is needed and so on.

As a first-order model to explain expanding rings, the two simplest dust geometries are a spherical shell and a planar slab, which are representative of circumstellar and interstellar media, respectively. Echoes from a spherical shell always appear concentric and can only grow as large as the shell's radius. For a slab, on the other hand, echoes should be observable to an arbitrary size, and will only appear as concentric, circular rings if the slab is aligned with the plane of the sky (an unlikely constraint).

Qualitatively, the echoes we observe are concentric at all epochs and end abruptly around 80 arcsec from S CrA, therefore the spherical shell model is already favored from these arguments, but for completeness we explore both the spherical-shell and the planar-slab geometries.

We identified the angular distance of each echo from S CrA (see Table 1), by iteratively fitting Gaussian functions to radial surface-brightness profiles taken at various position angles (Sugerman 2005), avoiding an "ear"-like filament which shows brightness variations over time. This "ear" feature is stationary and can be explained as an isolated knot with thickness smaller than $c \Delta t$, where $\Delta t$ is a typical pulse duration. An isolated knot in the direction perpendicular to the line of sight would only brighten and fade and no apparent motion would be seen. Therefore it seems that the ear-like feature is an isolated overdensity of much smaller size than the dust structure causing the expanding quasi-rings. Hence we excluded the ear-like signal from the analysis of the angular size of the expanding rings.

In order to compare the angular distance measurements with theoretical computations, guesses for the outburst times were inserted into the echo propagation equations for a spherical cloud model (Crause et al. 2005):

$\theta=\sqrt{2 z_{0} c t-(c t)^{2}} / d$

and a dust slab model (Tylenda 2004):

$\theta=\sqrt{(1+a)^{2}(c t)^{2}+2 z_{0} c t} / d$

where $\theta$ is angular radius, $d$ is distance from the observer to the star, $z_{o}$ is the distance from the star to the reflecting shell or slab, $a$ is the tangent of the perpendicular to the dust plane and the line of sight, $t$ is time from outburst and $c$ is the speed of light.

The outburst times together with $d$ and $z_{0}$ were iteratively fit by using a downhill simplex method (Press et al. 1992), in order to minimize the residuals of the angular radii measurements compared to those resulting from Eqs. (1) and (2). The results were refined via iteratively-reweighted least squares (Jefferys et al. 1987).

The spherical shell model places the star $121 \pm 29 \mathrm{pc}$ from earth, with the shell's average radius $9500 \pm 3800 \mathrm{AU}$, and the brightenings occurred on 9 July, 11 and 25 August, and 06 October 2007 (dates are uncertain by less than 1 day). Although the fit to the data is good $\left(\chi^{2}=0.7\right.$ per degree of freedom), Fig. 2 reveals that the echoes observed in September are 
A\&A 519, A7 (2010)

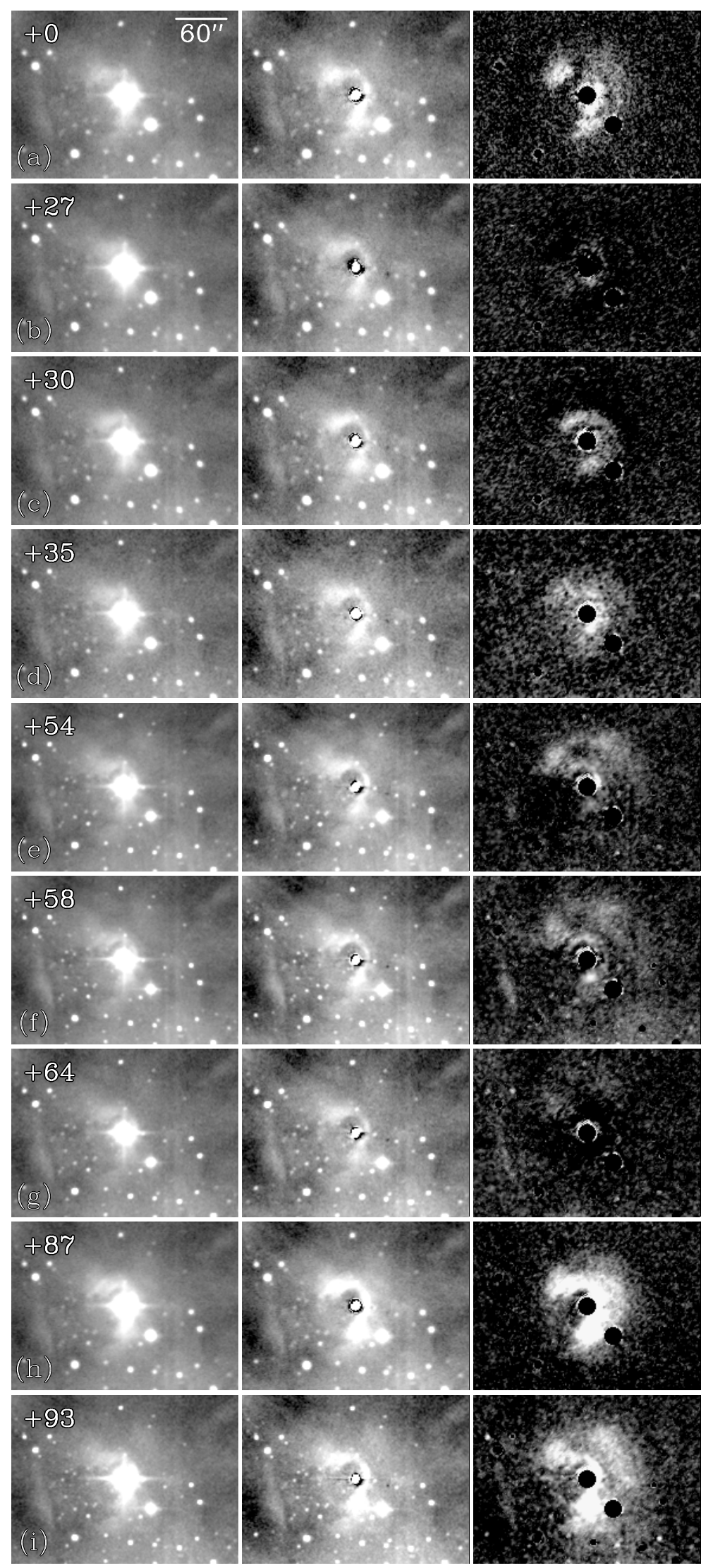

Fig. 2. Temporal sequences of images of S CrA, showing (left) direct images, (middle) direct images with the flux of S CrA removed via PSF subtraction, and (right) difference-only images. All panels are 270 by $200 \mathrm{arcsec}$, with north up, and east to the left. The number of days after the first epoch are indicated at top-left of each row. 


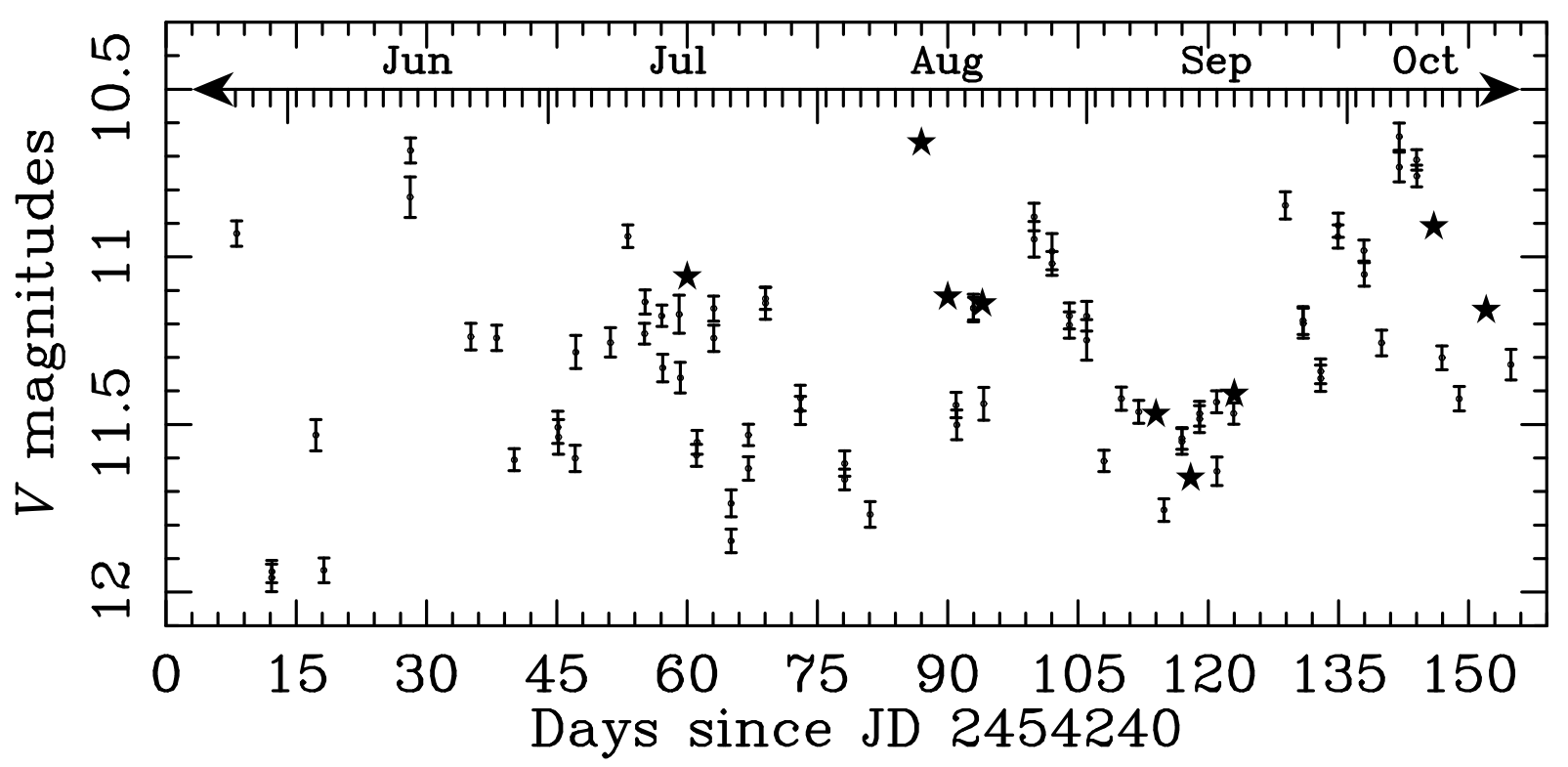

Fig. 3. $V$-band photometry of S CrA. Points with error bars show data from the All Sky Automated Survey (Pojmanski 1997) while our data from Table 1 are marked by stars.

very broad, up to 40 arcsec in angular width, requiring very long pulse durations and/or very thick dust. Light curves of S CrA from Fig. 3 and the past hundred years (Henden et al. 2009) indicate average brightening last 5-10 days. Consequently, to generate echoes as wide as are observed in September, the dust thickness (Sugerman 2003) would have to be equal to or greater than the dust's distance from the star. As this is an unlikely constraint, we instead consider whether the echoes observed in September may in fact be the convolution of light pulses from early and late August. Using the above brightening dates, the echoes observed in August would be 30 to 40 days old during the September epochs, placing them at angular radii of 65 to 75 arcsec, exactly where faint echoes are observed.

When deconvolved into two components, the echoes observed in September have angular radii of 45 and 63 arcsec (9 September), 52 and 67 arcsec (13 September), and 58 and 70 arcsec (18 September). With the spherical-shell model, these revised data yield a distance to $\mathrm{S} C r A$ of $138 \pm 16 \mathrm{pc}$, a shell radius of $10000 \pm 1700 \mathrm{AU}$, and new brightening dates of 8 July, 10 and 27 August, and 5 October 2007, with a $\chi^{2}$ per degree of freedom of 0.8 . That our model yields a distance to $\mathrm{S} \mathrm{CrA}$ consistent with previous values, and that the epochs of brightening agree with the photometric record (Fig. 3), confirm that this spherical-shell model is an appropriate first approximation of the actual dust geometry.

One might argue that echoes coming from a full spherical shell would be contracting rings after they reach their maximum radii and should should have been seen in our data. However, in order for contracting rings to be detectable, one would require a considerable amount of backscattering from the dust particles, which is inconsistent with the properties of the grains that we have derived (see next section). This is essentially based on the fact that the surface brightness of the echoes decreases with ring radii, which implies that the particles are not small and therefore cannot give rise to considerable backscattering. In conclusion, contracting rings would only be detectable under very special circumstances that are not met in our case.

Concerning the slab model, it yields two possible solutions. The first, with a $\chi^{2}$ per degree of freedom of 0.7 , places S CrA at a distance of $70 \pm 40 \mathrm{pc}$, with the dust inclined by $57^{\circ} \pm 7^{\circ}$ and lying $6300 \pm 4300 \mathrm{AU}$ in front of $\mathrm{S} \mathrm{CrA}$. The second model, with a $\chi^{2}$ per degree of freedom of 1.0, places S CrA beyond $1 \mathrm{kpc}$, with the dust oriented on the plane of the sky and located a few pc in front of the star. Although these fits are statistically good, the distances to S CrA are highly discrepant with other estimates. For the first, or close-slab model, "L"-type dust (see discussion section for its definition) explains many of the observed surface brightnesses if the dust density is roughly double that of the spherical shell model (see discussion section). However, it is difficult to explain the observed brightness of the largest-radii echoes, which are at very large scattering angles $\left(\theta>90^{\circ}\right)$, at which all dust types (not just "L"-type) are inefficient scatterers. In general, these echoes are observed to be two or more times brighter than those expected from dust with large scattering angles. Besides, the inclination by $57^{\circ} \pm 7^{\circ}$ would give rise to non concentric rings whose centers would be displaced several pixels and would therefore be easy to detect, but this was not the case. In the second, or distant-slab model, one expects very little change of surface brightness with time, since the echo light is essentially forward scattering at all times, however this is not observed. Given the slab models' inconsistencies with distance to the star and dust scattering properties, we consider that the slab does not appear to be a good approximation to the 3-dimensional dust structure and we will focus on the spherical or quasi spherical dust shell.

A plot showing the angular radii measured and the best fits is presented in Fig. 4

\section{Discussion}

An issue that might require some discussion is the fact that some echoes are fainter than others of similar age. This can be explained by the different intensity of the outbursts that give rise to the echoes. In fact, the farthest (and oldest) echoes from the star were only visible from the largest outburst (the one that took place around 10th of August 2007). Echoes as old as 40 days could only be seen from the August 10th outburst because it was extraordinarily bright (as is demonstrated in the light curve of 3) compared to the others. Echoes as old as these were not seen in 


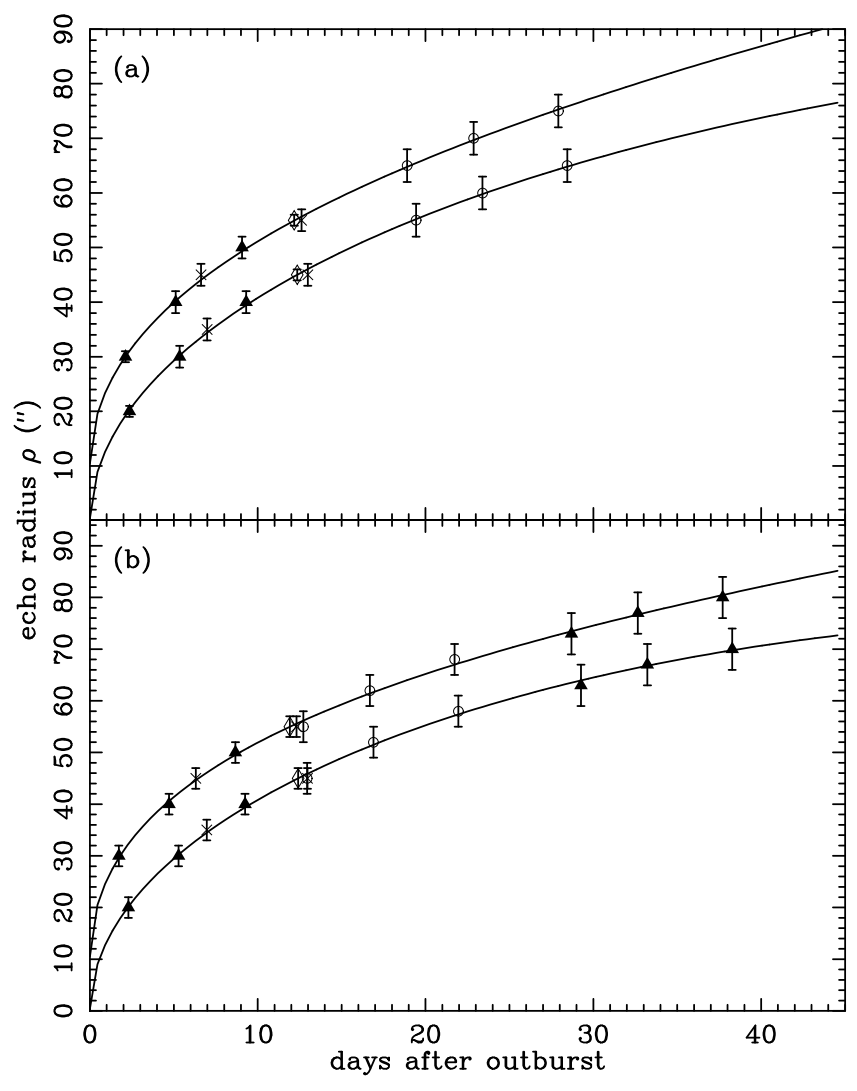

Fig. 4. Angular radii of the echoes observed around S CrA versus the time elapsed since the outbursts that formed them for a) the data in Table 1, and b) revised data assuming two echoes per epoch during September 2007. The slab model and its associated data are offset 10 arcsec above the spherical model in both panels. Diamonds denote echoes from July, triangles from August, circles from September, and crosses from October 2007.

our data from the other outbursts because a much more sensitive instrument would have been needed.

Although the echoes appear to have an overall circular or ring aspect, the flux is generally restricted to the northern half of the image, with a notable lack of echo flux toward the southeast. This could imply a lack of significant circumstellar dust to the south of S CrA, or perhaps interstellar dust could be selectively blocking the light from the echoes toward the south east direction. The existence of an outflow in exactly that direction was shown by Wang et al. (2004) and thus, it is possible that such outflow is sweeping any dust.

Therefore it must be stressed that the spherical shell that we propose as the best first order approximation cannot be a complete sphere because we do not see complete rings but arcs.

What is the origin of the dust? The most straightforward interpretation is that such dust is the remains of the stellar envelope, which would still be dense enough for a star as young as S CrA. Even though Carmona et al. (2007) quote an age for $\mathrm{S}$ CrA of around $2 \mathrm{Myr}$ and for that age the envelope and protoplanetary disk should have dissipated already, in their paper Carmona et al. (2007) implicitly admit that the resolution of their spectra is too low to detect the photospheric spectrum of this object. High resolution echelle spectra which show the photospheric spectrum (Appenzeller et al. 1986), give a lower photospheric temperature, which results in a lower age than estimated by Carmona et al. (2007). Most estimates result in $\sim 0.5 \mathrm{Myr}$ or lower. A young age is also indicated by the strong $\mathrm{mm}$ and sub-mm dust emission (Reipurth et al. 1993; Chini et al. 2003; Nutter et al. 2005; Juvela et al. 2009), the presence of an HH flow (HH 82) and the high and variable mass accretion rate (e.g. Walter \& Miner 2005). Nevertheless, the concentration of dust at $10^{4} \mathrm{AU}$ from the star is somewhat intriguing, and we therefore explore other more speculative possibilities.

A cloud of dust $10^{4} \mathrm{AU}$ from the star is close to the nominal distance of the Oort cloud from our Sun (Oort 1950). Although $\mathrm{S}$ CrA is a binary system, the primary star has a mass similar to the Sun (Carmona et al. 2007), which leads us to speculate that the scattering dust may be an Oort-cloud analog that is forming, or has formed at least partially around the star.

It is generally believed that the dust disks around young stars disappear when planets grow from accretion of the disk material. However, it might be possible that some dust material of the disk is not accreted but dynamically ejected by the gravitational effect of the forming planets or planetary embryos, if they are massive enough or if they grow quickly enough. Thus, planets or planetary embryos have likely formed already from the disk material of S CrA and therefore it appears possible that at least some dust from the disk may have been dynamically ejected to large perihelia and with a range of orbital inclinations.

Giant-planet formation is believed to require 1 to $10 \mathrm{Myr}$ (e.g. Rice \& Armitage 2003). With an estimated age of $\sim 0.5 \mathrm{Myr}$, S CrA may therefore be actively forming an extra-solar planetary system, but according to most models, the mass of the planetary embryos would still be far below the giant planet mass and therefore the proposed dynamical ejection scenario might not be effective enough.

As noted earlier in the observations and reductions section, the echoes do not appear as complete rings, which could signal an incomplete or inhomogeneous shell. This is a point in favor of the Oort-cloud-analog idea. Since the typical time for complete randomization of the orbital inclinations of the material ejected from a planetary disk is believed to be around 500 My (Duncan et al. 1987), one may not expect a fully spherical and complete Oort cloud from such a young star as S CrA. From Oort-cloud formation modeling such as the one in Duncan et al. (1987) the expected geometry of a young forming Oort cloud would be a torus rather than a sphere. Thus, another possible geometry worth of investigation for the dust around S CrA is that of a torus. We have briefly tested whether such model would be consistent with the data, in addition to the slab and spherical shell already mentioned. Figure 5 shows the regions of space probed by light echoes; in the first few weeks following a light pulse, echoes from a spherical shell will appear at all position angles, while those from a torus will only appear from the northern region. This leads us to propose that the echoing structure around S CrA might be compatible with an inclined torus. Unfortunately, the current dataset is insufficient to adequately perform detailed 3-dimensional geometric analysis, since the images are not homogenously sampled in time and the spatial resolution is not high.

Even though we are referring to the material that scatters the light pulses as "dust", from our observations we cannot determine whether the material is made of silicates or made of highly volatile condensates (which should be more properly referred to as "ice"). Because of the very low temperatures expected at around $10000 \mathrm{AU}$ from the star, condensates of highly volatile gases can exist, not only condensates of $\mathrm{H}_{2} \mathrm{O}$. We will continue to refer to the particles as "dust", but it must be pointed out that the exact composition of them cannot be revealed from our observations and therefore the existence of ice should be taken into account. 

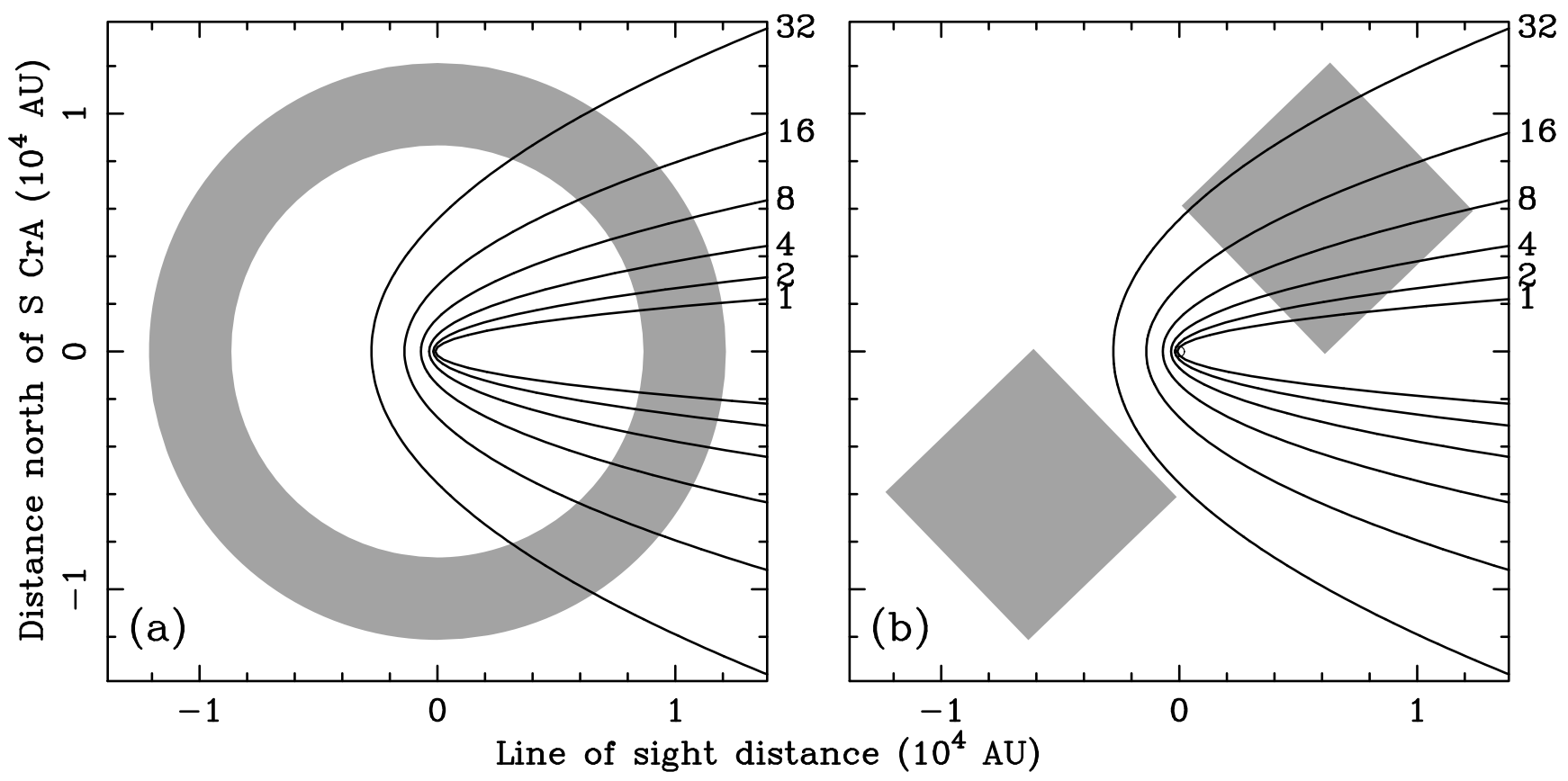

Fig. 5. Locations of echoes passing through a) a spherical shell and b) an inclined torus of dust. The number of days after maximum light is listed to the right of each parabola. The abscissa gives the distance in front of, or behind, the star, while the ordinate shows the distance above (north) or below (south) of the star.

Given that the star was only observed in one filter, detail modeling of the dust size distribution is not feasible, but constraints on the grain sizes can be derived from the data. If the dust were composed only of small grains (radius less than $0.01 \mu \mathrm{m}$ ), then the surface brightness of an echo from a spherical shell would present small changes with scattering angle. Since the echoes fade as they move away from $\mathrm{S} \mathrm{CrA}$, we conclude that the dust must include grains with radii $>0.1 \mu \mathrm{m}$. Indeed, dustscattering models from Sugerman (2003) suggest that some fraction of the dust must be large, i.e. $1 \mu \mathrm{m}$ or larger. Using a large-grain mixture (radii between $0.1-1.0 \mu \mathrm{m}$ ) of carbonaceous and silicate dust (referred to as "L"-type dust), we find the observed surface brightnesses are consistent with a total dust mass (for a complete spherical shell) of order $2 \times 10^{-3} M_{\odot}$ and this is likely an upper limit because the shell is not complete as we already emphasized. A similar mass is found when considering a cloud of ice particles. This mass is smaller than that in the minimum-mass solar nebula (Weidenschilling 1977), suggesting that the observed amount of dust around S CrA could have originated in its protoplanetary disk. Estimates of the mass of the current Oort cloud range from several to 100 Earth masses (Weissman 1983; Marochnik et al. 1988), which is considerably lower than $2 \times 10^{-3} M_{\odot}$, but one should keep in mind that the Oort cloud is not made of dust, but of larger bodies, as small grains are not expected to survive for billions of years due to non gravitational perturbations.

Alternative speculative explanations for the observed dust include mass ejections from earlier phases of the star's evolution. However, it is well known that protostellar mass ejections are generally bipolar, and since these cannot give rise to spheroidal or toroidal geometries, a shell at $10000 \mathrm{AU}$ would be a puzzling result from such ejections.

Perhaps a way to test whether these two alternative explanations are correct would be to measure the amount of gas that is present at $10000 \mathrm{AU}$. In the Oort cloud analog scenario one would expect no gas at all, whereas in the other two scenarios one would expect gas to dust ratios typical of the interstellar medium. Even though there is no concluding evidence in the literature, radio maps that trace $\mathrm{CO}$ gas show little or no gas around S CrA (Harju et al. 1993) whereas there is emission in the submillimetric and millimetric ranges coming from dust as already mentioned.

We therefore speculate that the dust producing the observed echoes is perhaps a young Oort-cloud analog, even though the most straightforward interpretation for the dust shell is the remains of S CrA's dust envelope, given S CrA's young age. As noted earlier, in order for a young Oort-cloud analog to form, the star system would require at least one massive planet forming inside the protoplanetary disk, to actively eject planetesimals and debris to larger orbital radii. In that case, an intriguing implication is that light echoes around young stellar systems could be used to indirectly detect the presence of jovian or massive planets.

\section{Conclusions}

We have detected not just one, but multiple echoes from light pulses of very young stars. Such echoes are an impressive document and indicate that similar echoes might be detectable around other young nearby stars or in star forming regions. This has good potential as a tool to study the environment around young stars and the process of planet formation in those systems.

The echoes surrounding S CrA are more confined and are much easier to interpret than those coming from $\mathrm{R} \mathrm{CrA}$ although the latter are more intense. We have presented a preliminary analysis of the echoes surrounding S CrA. From the information contained in the angular distance of the echoes versus time from outbursts we have been able to derive basic properties of the dust that is scattering the light-pulses. The dust must be concentrated in a region around $10000 \mathrm{AU}$ from the star and the best geometry of the dust in terms of reproducing the observations is an incomplete spherical shell or an inclined torus; a planar slab geometry 
appears to be ruled out by the observations, but it would require dust at a similar distance in order of magnitude. The best fit to the echo radii gives a distance from Earth to $\mathrm{S} \mathrm{CrA}$ of $128 \pm 16 \mathrm{pc}$ in the case of the spherical shell distribution, which is consistent with previous rough estimations of the distance to $\mathrm{S} \mathrm{CrA}$ (see the distance discussion in Neuhäuser \& Forbrich 2008) whereas the dust slab geometry gives a distance to $\mathrm{S} \mathrm{CrA} \mathrm{that} \mathrm{cannot} \mathrm{be}$ reconciled with other observations in the literature. The derived distance implies that these are the closest echoes to the Earth that have ever been recorded.

Concerning the information extracted from the surface brightness of the echoes, the mass of the dust shell required in order to reproduce the observed echoes surface brightness is in the order of $2 \times 10^{-3} M_{\odot}$ and must be in the form of particles larger than $0.1 \mu \mathrm{m}$ to explain the fading of the echoes as they grow in angular size. Such dust structure is probably the remnant of the envelope typical of very young stars. But because of the concentration near $10000 \mathrm{AU}$, we speculate that the dust particles might have been dynamically ejected from the star's disk through the action of forming planets and ended up where they are now in a similar way as planetesimals have been ejected from the solar system to form the Oort cloud.

We must caution the reader that we have presented just a first order model to explain the echoes around S CrA. A well sampled lightcurve of SCrA and high spatial resolution images would be needed in order to derive the detailed 3-dimensional structure of the dust surrounding S CrA.

Acknowledgements. We are grateful to M. Fernández, M. Osorio and G. Anglada for very helpful discussions. This research was partially supported by Spanish grants AYA2005-07808-C03-01, PCI2005-A7-0180, AYA2008-06202C03-01, P07-FQM-02998 and European FEDER funds. B.E.K.S. was supported, in part, by HST grant GO-10607. We acknowledge with thanks the variable star observations from the AAVSO International Database contributed by observers worldwide and used in this research. We also acknowledge the use of ASAS database. R.D. acknowledges financial support from the MICINN (contract Ramón y Cajal). We also acknowledge the fruitful comments from the anonymous referee.

\section{On-line material}

The two animations cited in the text are also available at:

http://www.iaa.es/ ortiz/animacion1.avi

http://www .iaa.es/ ortiz/S-animation.gif

\section{References}

Appenzeller, I., Jetter, R., \& Jankovics, I. 1986, A\&AS, 64, 65 Barnard, E. E. 1895, MNRAS, 55, 442
Bellingham, J. G., \& Rossano, G. S. 1980, AJ, 85, 555

Bode, M. F., \& Evans, A. 1985, A\&A, 151, 452

Boffi, F. R., Sparks, W. B., \& Macchetto, F. D. 1999, A\&AS, 138, 253

Bond, H. E., \& Sparks, W. B. 2009, A\&A, 495, 371

Bond, H. E., Henden, A., Levay, Z. G., et al. 2003, Nature, 422, 405 Cappellaro, E., Patat, F., Mazzali, P. A., et al. 2001, ApJ, 549, L215

Carmona, A., van den Ancker, M. E., \& Henning, T. 2007, A\&A, 464, 687

Chini, R., Kämpgen, K., Reipurth, B., et al. 2003, A\&A, 409, 235

Couderc, P. 1939, Ann. Astrophys., 2, 271

Crause, L. A., Lawson, W. A., Menzies, J. W., \& Marang, F. 2005, MNRAS, 358,1352

Crotts, A. P. S., \& Yourdon, D. 2008, ApJ, 689, 1186

Duncan, M., Quinn, T., \& Tremaine, S. 1987, AJ, 94, 1330

Gaidos, E. J. 1994, Icarus, 109, 382

Graham, J. A., \& Phillips, A. C. 1987, PASP, 99, 91

Harju, J., Haikala, L. K., Mattila, K., et al. 1993, A\&A, 278, 569

Henden, A. A., Welch, D. L., Terrell, D., \& Levine, S. E. 2009, in Am. Astron. Soc. Meeting Abstracts, 214, 407.02

Hubble, E. 1921, Mt. Wilson Ann. Rep., 20, 252

Jefferys, W. H., Fitzpatrick, M. J., \& McArthur, B. E. 1987, Celest. Mech., 41, 39

Juvela, M., Pelkonen, V., \& Porceddu, S. 2009, A\&A, 505, 663

Krause, O., Rieke, G. H., Birkmann, S. M., et al. 2005, Science, 308, 1604

Lightfoot, J. F. 1989, MNRAS, 239, 665

Liu, J., Bregman, J. N., \& Seitzer, P. 2003, ApJ, 582, 919

Marochnik, L. S., Mukhin, L. M., \& Sagdeev, R. Z. 1988, Science, 242, 547

Marraco, H. G., \& Rydgren, A. E. 1981, AJ, 86, 62

Neuhäuser, R., \& Forbrich, J. 2008, The Corona Australis Star Forming Region, ed. B. Reipurth, 735

Newman, A. B., \& Rest, A. 2006, PASP, 118, 1484

Nutter, D. J., Ward-Thompson, D., \& André, P. 2005, MNRAS, 357, 975

Oort, J. H. 1950, Bull. Astron. Inst. Netherlands, 11, 91

Pojmanski, G. 1997, Acta Astron., 47, 467

Press, W. H., Teukolsky, S. A., Vetterling, W. T., \& Flannery, B. P. 1992, Numerical recipes in FORTRAN, The art of scientific computing, ed. W. H. Press, S. A. Teukolsky, W. T. Vetterling, \& B. P. Flannery

Quinn, J. L., Garnavich, P. M., Li, W., et al. 2006, ApJ, 652, 512

Reipurth, B., Chini, R., Krugel, E., Kreysa, E., \& Sievers, A. 1993, A\&A, 273, 221

Rest, A., Suntzeff, N. B., Olsen, K., et al. 2005, Nature, 438, 1132

Rest, A., Matheson, T., Blondin, S., et al. 2008a, ApJ, 680, 1137

Rest, A., Welch, D. L., Suntzeff, N. B., et al. 2008b, ApJ, 681, L81

Rice, W. K. M., \& Armitage, P. J. 2003, ApJ, 598, L55

Sparks, W. B., Macchetto, F., Panagia, N., et al. 1999, ApJ, 523, 585

Sparks, W. B., Bond, H. E., Cracraft, M., et al. 2008, AJ, 135, 605

Stetson, P. B. 1987, PASP, 99, 191

Struve, O. 1862, MNRAS, 22, 242

Sugerman, B. E. K. 2003, AJ, 126, 1939

Sugerman, B. E. K. 2005, ApJ, 632, L17

Suntzeff, N. B., Heathcote, S., Weller, W. G., Caldwell, N., \& Huchra, J. P. 1988, Nature, 334, 135

Tomaney, A. B., \& Crotts, A. P. S. 1996, AJ, 112, 2872

Tylenda, R. 2004, A\&A, 414, 223

Walter, F. M., \& Miner, J. 2005, in 13th Cambridge Workshop on Cool Stars, Stellar Systems and the Sun, ed. F. Favata, G. A. J. Hussain, \& B. Battrick, ESA SP, 560, 1021

Wang, H., Mundt, R., Henning, T., \& Apai, D. 2004, ApJ, 617, 1191

Weidenschilling, S. J. 1977, Ap\&SS, 51, 153

Weissman, P. R. 1983, A\&A, 118, 90

Zacharias, N., Urban, S. E., Zacharias, M. I., et al. 2004, AJ, 127, 3043 